\title{
Replacement of Adenovirus Type 5 Fiber Shaft Heparan Sulfate Proteoglycan-Binding Domain with RGD for Improved Tumor Infectivity and Targeting
}

\author{
Neus Bayo-Puxan, Marta Gimenez-Alejandre, Sergio Lavilla-Alonso, ${ }^{2}$ Alena Gros, \\ Manel Cascallo, Akseli Hemminki, ${ }^{2}$ and Ramon Alemany ${ }^{1}$
}

\begin{abstract}
Tumor targeting on systemic adenovirus administration is key to improve the prospects of adenovirus-mediated gene therapy and virus therapy of cancer. Despite many genetic and ligand conjugation approaches this objective remains elusive. Ablation of human adenovirus type 5 (Ad5) binding to its natural receptors in airway epithelial cells, that is, the coxsackievirus and adenovirus receptor (CAR) and integrins, does not impact on the preeminent liver tropism of adenovirus in the bloodstream. This is explained by a distinct entry pathway mediated by blood factors and heparan sulfates. Mutation of the KKTK heparin sulfate-binding domain of the fiber shaft to GATK results in liver transduction detargeting, but it is not compatible with otherwise useful HIloop tumor-targeting ligand insertions such as the insertion of Arg-Gly-Asp (RGD). To circumvent this problem we have mutated the KKTK domain to RGDK, and analyzed the liver-detargeting and tumor-targeting transduction properties of this replacement mutant. Similar to RGD at the HI-loop, RGD at this new shaft location efficiently enhances the infectivity of adenovirus and improves the tumor-to-liver transduction ratio in vivo.
\end{abstract}

\section{Introduction}

$\mathbf{T}$ REATMENT SUCCESS in cancer virotherapy and gene therapy depends on the ability of viruses and vectors to reach disseminated cancer cells on systemic administration.

To retarget adenovirus serotype 5 (Ad5) to disseminated tumor cells it is necessary to abrogate liver transduction (liver detargeting) and to expose new ligands specific for tumor cells on capsid proteins (tumor targeting). The Ad5 fiber knob domain binds to the primary coxsackievirus and adenovirus receptor (CAR) (Bergelson et al., 1997) to initiate infection and the Arg-Gly-Asp (RGD) motif of the penton base interacts with cellular integrins $\alpha_{\mathrm{v}} \beta_{3}$ and $\alpha_{\mathrm{v}} \beta_{5}$ to promoter internalization (Wickham et al., 1993; Nemerow and Stewart, 1999). Low-affinity interactions with heparan sulfate proteoglycans (HSPGs) can also mediate Ad5 cell entry. The ${ }^{91} \mathrm{KKTK}^{94}$ domain of the fiber shaft domain has been postulated as the HSPG-binding domain (Dechecchi et al., 2000, 2001). In vitro competition analysis with heparin supports a major role of HSPGs in Ad5 entry in the absence of CAR
(Smith et al., 2002, 2003b; Bayo-Puxan et al., 2006). The KKTK domain contributes to Ad5 liver transduction in vivo, as its mutation to GATK or GAGA detargets liver transduction (Smith et al., 2003a,b; Nicol et al., 2004; Bayo-Puxan et al., 2006; Kritz et al., 2007; Rittner et al., 2007).

Despite the fact that Ad5 uses CAR and integrins during the life cycle to infect airway epithelial cells, the double ablation of CAR and integrin binding fails to reduce hepatocyte transduction in vivo when the virus is injected into the bloodstream (Alemany and Curiel, 2001; Einfeld et al., 2001; Martin et al., 2003; Smith et al., 2003b; Bayo-Puxan et al., 2006). This can be explained by the role of blood factors in adenovirus liver transduction (Shayakhmetov et al., 2005b; Parker et al., 2006, 2007; Baker et al., 2007). Human coagulation factor IX (FIX) and complement C4-binding protein (C4BP) bridge the fiber knob to the HSPGs and low-density lipoprotein receptor-related protein (LRP) of the hepatocyte, whereas coagulation factor $X(F X)$ binds to hypervariable regions (HVRs) of the hexon protein (Kalyuzhniy et al., 2008; Vigant et al., 2008; Waddington et al., 2008).

\footnotetext{
${ }^{1}$ Translational Research Laboratory, Institut d'Investigació Biomèdica de Bellvitge (IDIBELL)-Institut Català d'Oncologia, L'Hospitalet de Llobregat, 08907 Barcelona, Spain.

${ }^{2}$ University of Helsinki, Cancer Gene Therapy Group, Molecular Cancer Biology Program, and Haartman Institute and Finnish Institute for Molecular Medicine, 00290 Helsinki, Finland.
} 
The HI-loop of the fiber knob domain has been used to accommodate tumor-selective peptides conferring new entry pathways to recombinant adenoviral vectors (Dmitriev et al., 1998; Xia et al., 2000; Mizuguchi et al., 2001). However, several attempts to retarget adenoviral vectors by ligand insertion into the HI-loop when the fiber shaft is mutated have failed. The insertion of selective peptides such as QPE (Kritz et al., 2007), HEW (Rittner et al., 2007), and RGD into the HIloop when the KKTK domain was mutated to GATK or GAGA did not rescue the infectivity of the detargeted vectors (Bayo-Puxan et al., 2006; Kritz et al., 2007). A possible explanation of this nonfunctional HI-loop in a shaft-mutated fiber is that the KKTK mutation affects the bending or structure of the fiber, precluding proper function of the fiber knob.

We hypothesize that insertion of tumor-selective ligands at capsid locations other than the knob could be more compatible with the KKTK shaft mutation and may allow retargeting of shaft-mutated adenoviral vectors to disseminated cancer cells. Still, among the various capsid proteins the fiber seems to be the best location for ligand insertion as it detaches from the capsid soon after cell entry. To explore a location in the fiber different from the HI-loop and compatible with the shaft mutation, we replaced the KKTK domain directly with RGD. RGD at the shaft KKTK domain increased tumor cell transduction and improved the tumor-toliver ratio in vivo of adenoviral vectors.

\section{Materials and Methods}

\section{Adenoviral vectors}

All vectors carried the green fluorescent protein (GFP)encoding gene and a luciferase expression cassette under the control of the cytomegalovirus (CMV) promoter, replacing the E1 region. Vectors AdGFPLuc, AdTLattP, AdTLRGD, AdTLG, and AdTLGR have been described previously (Alemany and Curiel, 2001; Bayo-Puxan et al., 2006).

The HSPG-binding KKTK domain of the fiber shaft was replaced with RGDK, using polymerase chain reaction (PCR)-based mutagenesis of pBSattB. This shuttle plasmid carries the wild-type Ad5 fiber gene flanked by attB donor sequences for site-specific homologous recombination and by EcoRI. The oligonucleotides (oligos) used were KKT-RGD (5'CCAAAATGTAACCACTGTGAGCCCACCTCTCCGAGGA GACAAGTCAAACATAAACCTGGA-3'), which contains the RGDK mutation, and Fiber Down5 (5'-GGTTACATTTT GGGAGGTAA-3'). Ad5-modified fiber RGDK was digested with EcoRI, gel purified, and self-ligated to obtain a DNA circle that contains the RGDK fiber preceded by the attB sequence (attB-FiberRGDK). AdTLRGDK was generated on $293 \Phi C 31$ cells by site-specific homologous recombination. The attB-FiberRGDK circle was transfected into 293ФC31 cells that were previously infected with fiberless Ad5 vector AdTLattP (fiber open reading frame [ORF] replaced with the acceptor attP sequence). AdTLRGDK was then plaque purified and propagated on 293 cells. This $\Phi C 31$-mediated integration represents a novel system to efficiently obtain viruses with recombinant fibers. To quantify the protein content of purified adenoviral vectors, proteins of purified virions were denatured by boiling and separated by sodium dodecyl sulfate-polyacrylamide gel electrophoresis (SDS-PAGE) and then stained with silver nitrate.
Ad5 vectors were purified by $\mathrm{CsCl}$ gradients and dialyzed in phosphate-buffered saline (PBS)-HCl-magnesium with $5 \%$ glycerol (Invitrogen, Carlsbad, CA). The particle titer was determined by optical density (OD) and all mutations were confirmed by sequencing the purified viral DNA.

\section{Cell lines}

HEK-293 (293), BxPC-3, BZR, L929, SkMel-28, HT116, HT29, Co115, SW620, SW480, and CaCo-2 cancer cell lines were obtained from the American Type Culture Collection (ATCC, Manassas, VA). $293 \Phi C 31$ cells were kindly provided by M. Chillon (Centre de Biotecnologia Animal i de Teràpia Gènica [CBATEG], Universitat Autònoma de Barcelona [UAB], Barcelona, Spain). Cell lines were maintained in Dulbecco's modified Eagle's medium (DMEM) (GIBCO; Invitrogen) containing $5 \%$ fetal bovine serum (GIBCO; Invitrogen) and penicillin-streptomycin (GIBCO; Invitrogen). 293ФC31 cells were maintained with hygromycin at $250 \mu \mathrm{g} / \mathrm{ml}$ for selection (GIBCO; Invitrogen).

\section{Adenovirus-mediated transduction assays}

One day before infection, 20,000 HEK-293, BxPC-3, BZR, SkMel-28, HT116, HT29, Co115, SW620, SW480, and CaCo-2 cells and 40,000 L929 cells were seeded per well in 96-well plates. Virus was added at the number of viral particles (VP) per cell indicated in the figure legends in $60 \mu \mathrm{l}$ of growth medium and incubated for $20 \mathrm{hr}$ for 293 cells and for $48 \mathrm{hr}$ for the other cell types. To quantify luciferase activity and protein content, cells were processed as previously described (Bayo-Puxan et al., 2006). Results are expressed as relative light units (RLU) per microgram of protein.

\section{Biodistribution of adenoviral vectors}

Recombinant Ad5 vectors were injected via the tail vein into C57BL/ 6 mice $\left(n=5,5 \times 10^{10} \mathrm{VP} /\right.$ mouse). Animals were kept and manipulated in accordance with recommendations of the Federation of European Laboratory Animal Science Associations for the proper use of laboratory animals. Three days after injection, organs and tissues were harvested and snap-frozen. Biodistribution of adenoviral vectors in tissues was determined as previously described (Bayo-Puxan et al., 2006). Results are shown as relative light units per milligram of protein. The Mann-Whitney test was used to determine whether differences among groups were statistically significant.

\section{Subcutaneous tumor xenograft model in nude mice}

Eight million BxPC-3 cells were xenografted under the skin of each flank in BALB/c $n u / n u$ mice. When the nodules reached approximately $80 \mathrm{~mm}^{3}$ a single dose of $5 \times 10^{10} \mathrm{VP}$ of each recombinant vector was intravenously administered into the mice $(n=5)$. Biodistribution of adenoviral vectors in tissues was determined as described previously. Ratios of transduction levels between liver and tumors were then calculated for each tumor and scaled, using a $\log _{10}$ transformation, and transduction relative to the liver (also on a $\log$ scale) was obtained for the two tumors of each mouse. The Mann-Whitney test was used to determine whether differences among groups were statistically significant. 
a

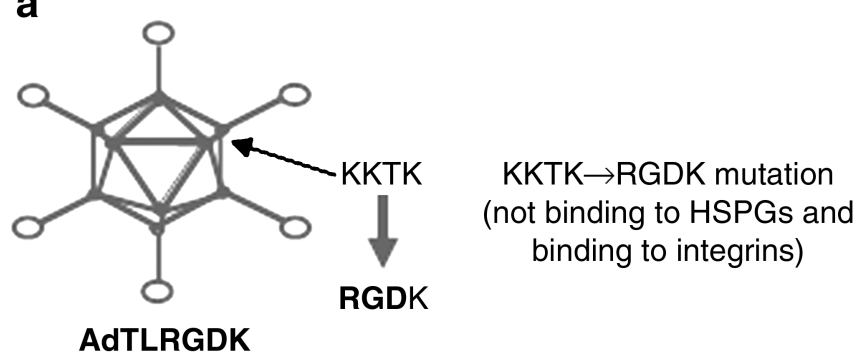

b
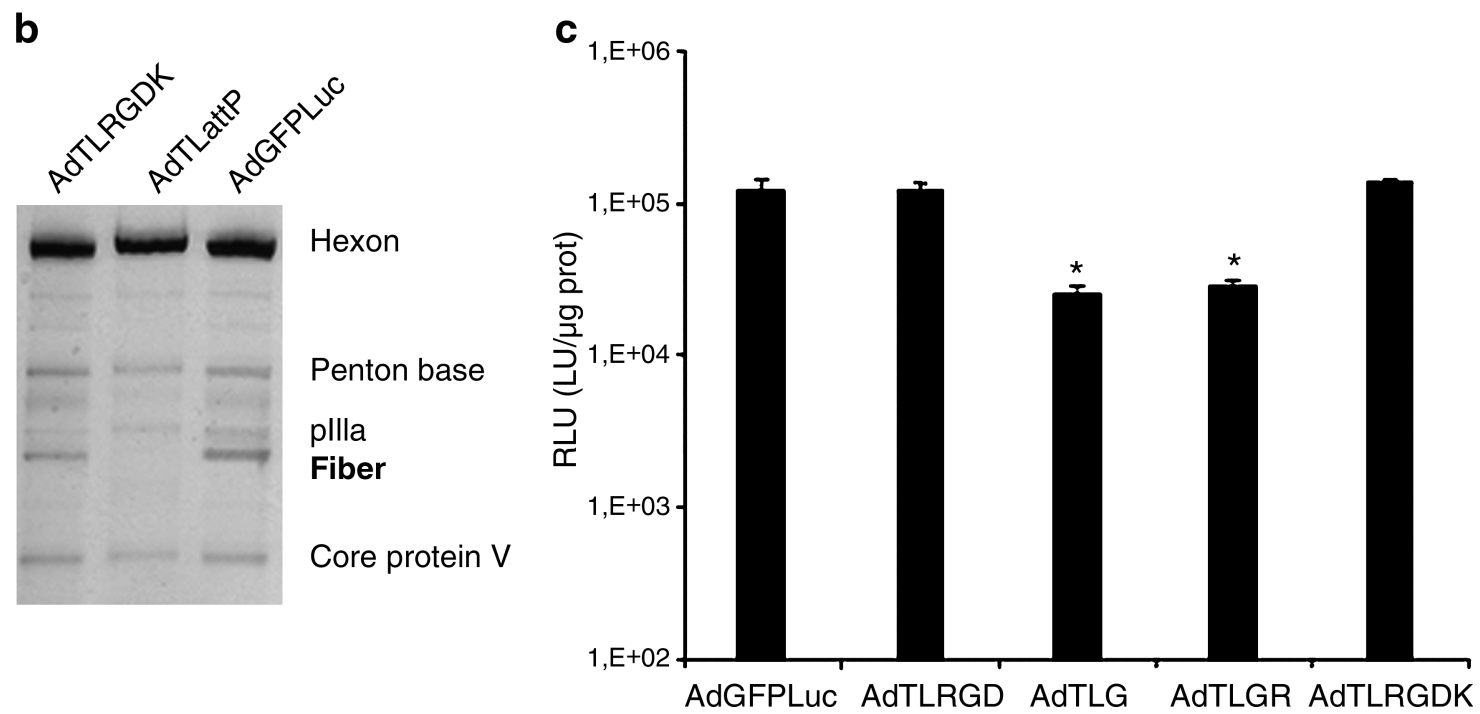

FIG. 1. Characterization of AdTLRGDK. (a) Schematic representation of fiber shaft mutation KKTK $\rightarrow$ RGDK of AdTLRGDK. (b) Protein content of purified virus. About $8 \times 10^{9}$ VP of purified virus was boiled and separated by SDS-PAGE and then stained with silver nitrate. AdTLattP was used as a control. (c) Infectivity assay on 293 cells. 293 cells $\left(22.5 \times 10^{4}\right)$ were infected for $1 \mathrm{hr}$ at $4^{\circ} \mathrm{C}$ with AdGFPLuc, AdTLRGD, AdTLG, AdTLGR, or AdTLRGDK at 100 VP/cell. Luciferase activity was measured and protein content was determined $24 \mathrm{hr}$ after infection. Results are shown as relative light units (RLU $[\mu$ g protein $]-1) .{ }^{*} p \leq 0.05$, significantly different from AdGFPLuc (Mann-Whitney test).

\section{Results}

\section{Description and properties of recombinant adenoviral vectors with fiber shaft HSG-binding domain replaced with $R G D$}

Taking into account our previous results (Bayo-Puxan et al., 2006), we have explored the possibility to increase Ad5 tumor infectivity and at the same time retain the liverdetargeting properties of fiber shaft mutation. With this aim we replaced the amino acids ${ }^{91} \mathrm{KKT}^{93}$, located in the fiber shaft domain, with the tumor-selective RGD tripeptide. Because the KKTK domain interacts with HSPGs, an RGD insertion at this domain should be exposed to recognize cellular integrins. The mutation KKTK $\rightarrow$ RGDK was introduced in a wild-type fiber background (Fig. 1a). Contrary to shaft-mutated adenoviral vectors described previously
(AdTLG and AdTLGR), AdTLRGDK could be propagated in 293 cells as efficiently as the nonmodified vector AdGFPLuc. Moreover, the protein content of purified vectors showed that the shaft mutation KKTK $\rightarrow$ RGDK did not reduce the assembly of fibers into the virion capsid, implying a correct trimerization (Fig. 1b). To confirm this and to better know the differences in transduction efficiency between the fiber shaft-mutated vectors, we performed a transduction assay on 293 cells (Fig. 1c). As we previously observed, the GATK fiber shaft-mutated vector AdTLG, and a similar vector but with RGD at the HI-loop of the knob (AdTLGR), transduced 293 cells inefficiently (10-fold less efficient compared with unmodified AdGFPLuc). This result correlates with the need to use 211B cells (fiber-complementing cells derived from 293 cells) to produce these shaft-mutated vectors. On the other hand, the shaft-retargeted adenoviral vector AdTLRGDK

FIG. 2. Transduction properties of vectors with RGD in the fiber shaft compared with adenoviral vectors containing RGD in the HI-loop of shaft-mutated fibers in (a) CaCo-2, (b) SW620, (c) HT29, (d) HTC116, (e) Co115, (f) SW480, (g) L929, and (h) BXPC-3 cells. Cells were infected with the viral particles per cell indicated for each vector: AdGFPLuc, AdTLG, AdTLGR, and AdTLRGDK. Luciferase activity and protein content were quantified $48 \mathrm{hr}$ after infection. Results are shown as mean relative light units (RLU $[\mu \mathrm{g}$ protein] -1$)(n=4) \pm \mathrm{SD}$ (error bars). 
a

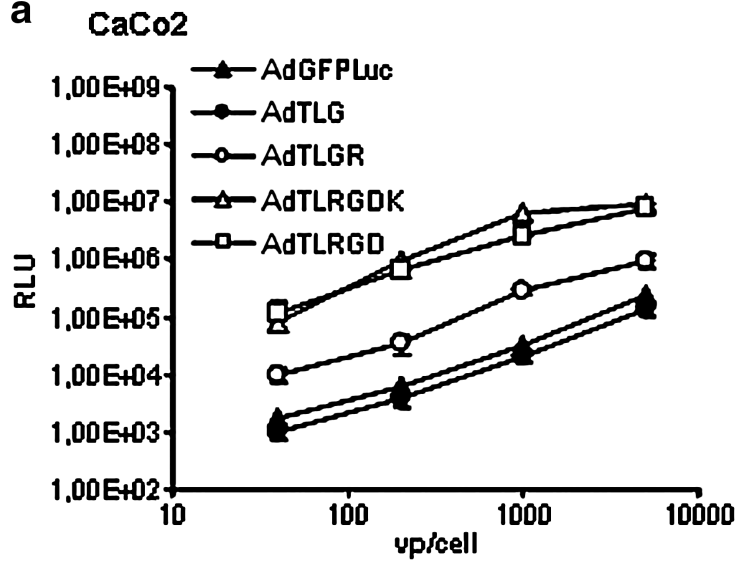

C

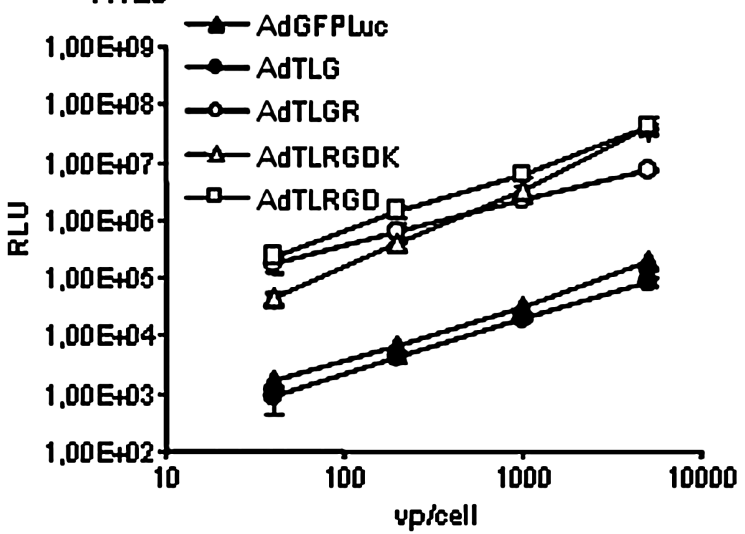

e

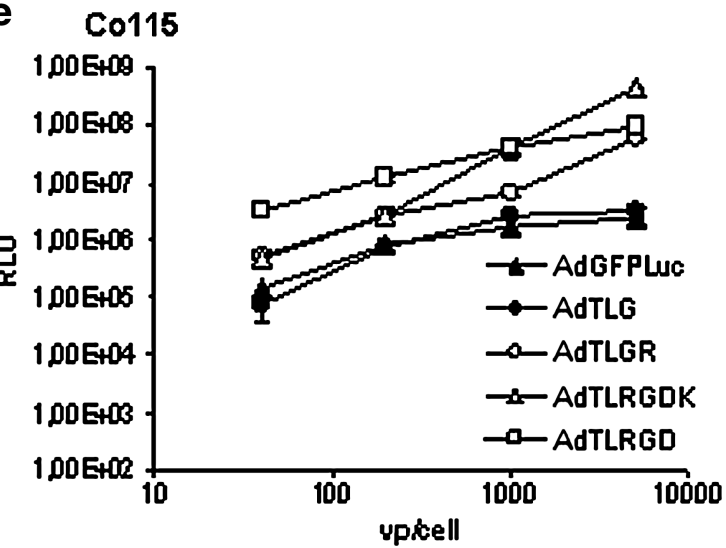

g $\quad$ L929

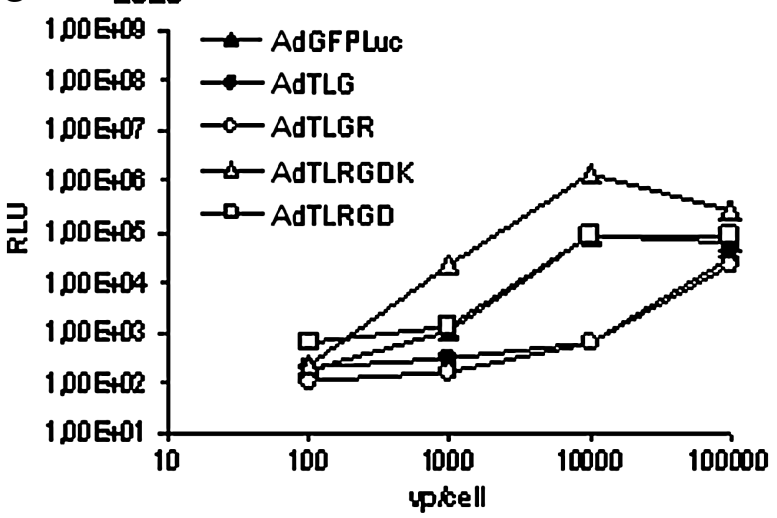

b sw620

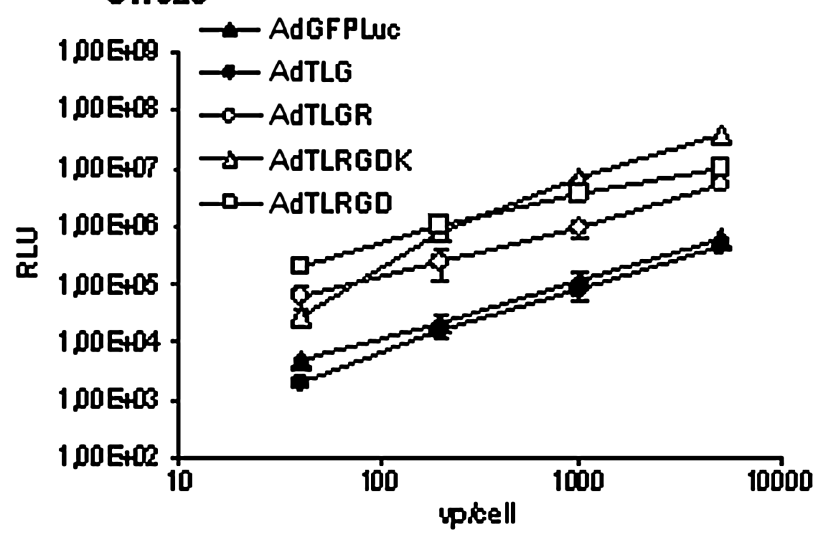

d HTC116
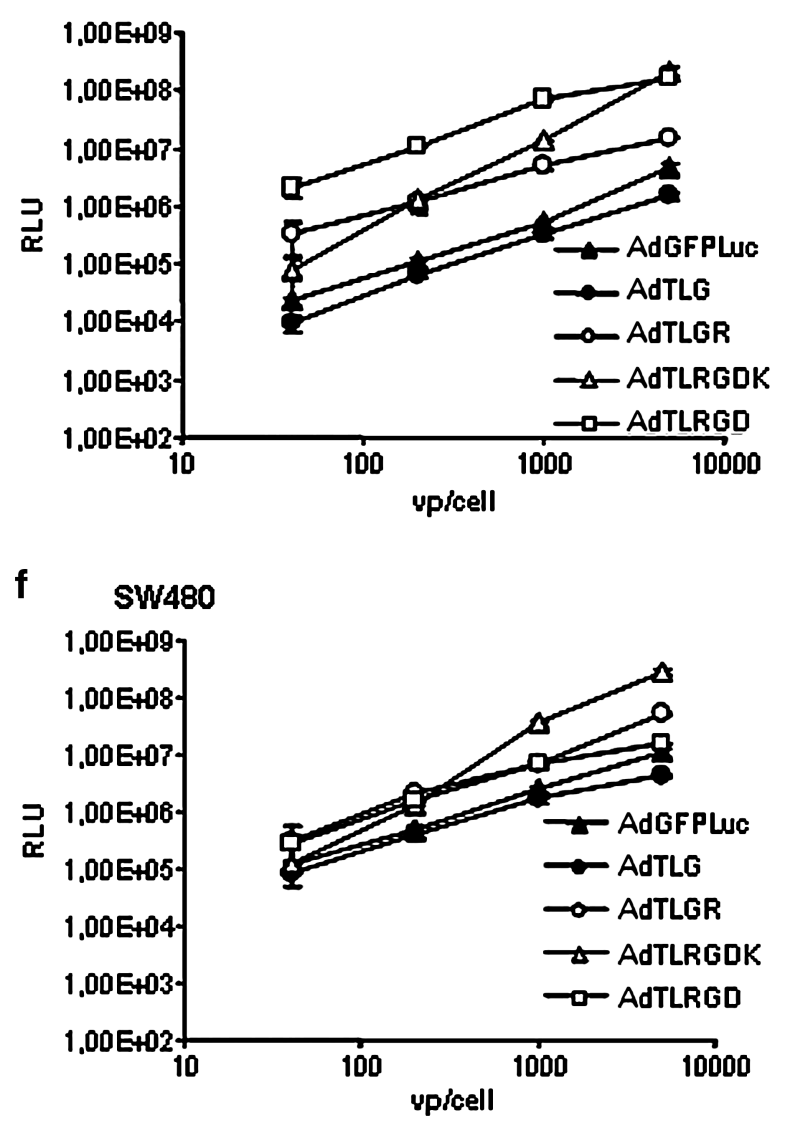

h $\mathrm{BxPC} 3$

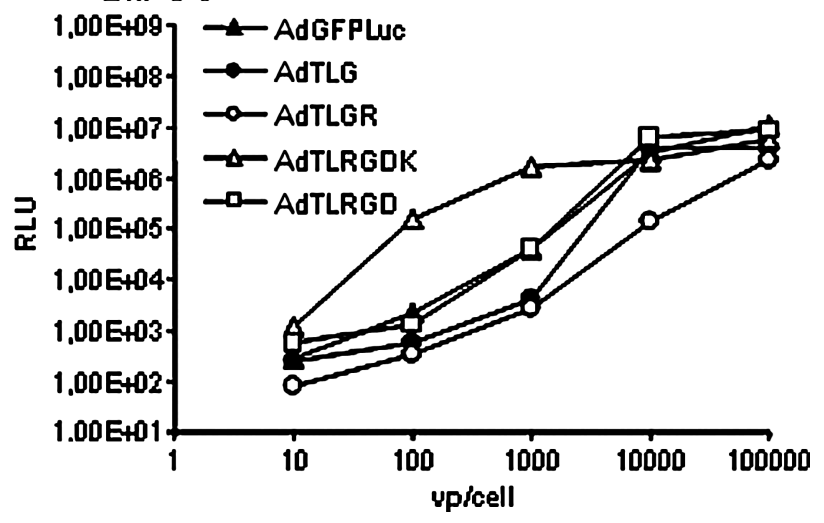




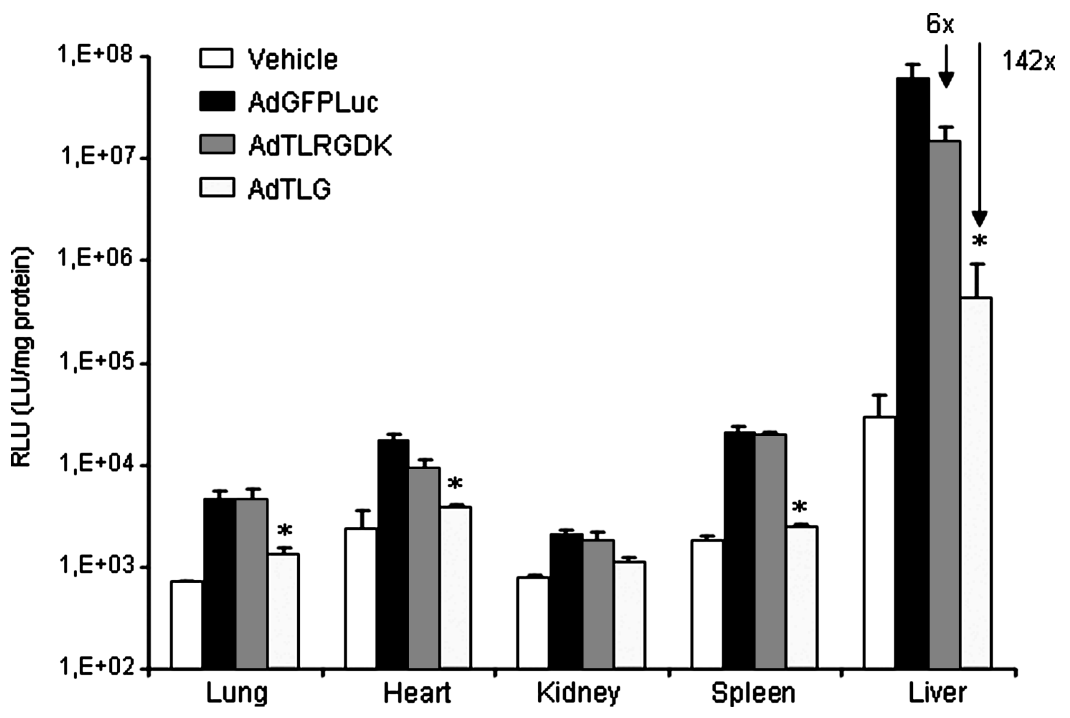

FIG. 3. Biodistribution of luciferase expression in mice after systemic administration of fiber shaft-retargeted vector AdTLRGDK compared with nonmodified vector AdGFPLuc and fiber shaft-mutated vector AdTLG. About $5 \times 10^{10}$ VP of the corresponding vector was injected intravenously into C57BL/6 mice. Two days later, tissues were harvested and luciferase activity and protein were quantified, as indicated in Materials and Methods. Results are shown as RLU [relative light units (mg protein) -1$]$. ${ }^{*} p \leq 0.05$, significantly different from AdGFPLuc.

transduced 293 cells as efficiently as the nonmodified vector AdGFPLuc and the adenoviral vector with RGD insertion into the HI-loop in a wild-type fiber background, AdTLRGD.

\section{$R G D$ at fiber shaft mediates enhanced Ad5 infectivity in cancer cell lines}

To know whether the insertion of RGD in a new location of the fiber may recognize integrins and increase transduction efficiency, we performed a transduction assay on a panel of cancer cell lines. The transduction properties of adenoviral vectors with RGD replacing the KKTK domain (AdTLRGDK) or with RGD inserted at the HI-loop in both a shaft-mutated background (AdTLGR) and a wild-type fiber background (AdTLRGD) were compared (Fig. 2). AdTLRGDK transduced all cell lines more efficiently than the adenoviral vector with the RGD insertion at the HI-loop in a shaft-mutated background (AdTLGR) and the nonmodified vector AdGFPLuc. In a shaft-mutated background, RGD mediated better transduction when located at the KKTK site than at the HI-loop (AdTLRGDK vs. AdTLGR). AdTLRGDK transduced SW480 and SW620 cells as much as AdTLRGD did, but AdTLRGDK transduced Co115 and CaCo-2 cells better than AdTLRGD. In L929 cells, which express a low level of CAR, AdTLRGDK increased transduction to a higher level compared with all other vectors. We concluded therefore that the substitution of KKT by RGD allowed recognition of integrins and infection of L929 cells. Of note, RGD increased Ad5 infectivity when located at the shaft but not the HI-loop of a shaft-mutated fiber (AdTLRGDK vs. AdTLGR). This indicates that the shaft mutation GATK precludes proper exposure of RGD into the HI-loop, according our previous results (Bayo-Puxan et al., 2006), and points out the potential of the KKTK domain of the Ad5 shaft as an alternative locus to insert tumor-selective peptides in a KKTK mutant detargeted Ad5 fiber background.

\section{AdTLRGDK transduction in vivo}

Taking into account that the shaft mutation GATK reduced liver transduction by 140-fold in mice (Bayo-Puxan et al., 2006), we evaluated the role of the RGDK mutation in liver transduction. We injected $5 \times 10^{10} \mathrm{VP}$ of each vector (unmodified vector AdGFPLuc, shaft-retargeted vector AdTLRGDK, and shaft-mutated vector AdTLG) into C57BL/6 mice via the tail vein. The biodistribution of gene expression was quantified in tissue cell extracts (Fig. 3). As we have previously shown, luciferase quantification revealed a different biodistribution profile for adenoviral vector carrying the shaft mutation KKTK to GATK (AdTLG). In contrast, the adenoviral vector carrying the shaft mutation KKTK to RGDK (AdTLRGDK) did not change significantly the transduction pattern of Ad5 in mice compared with a nonmodified vector (AdGFPLuc). The shaft mutation KKTK to RGDK reduced the levels of liver detargeting previously observed with the GATK shaft-mutated adenoviral vector. AdTLRGDK partially reduced (6-fold) liver transduction compared with the nonmodified vector AdGFPLuc, but the difference was not statistically significant $(p>0.05)$. We concluded that the RGDK mutation reduced the level of liver detargeting compared with the GATK mutation (AdTLG).

\section{RGD substitution of the HSG-binding domain increases tumor-to-liver ratio}

Despite the fact that AdTLRGDK did not show liver detargeting at the same level as AdTLG, our observation that RGD located at the shaft domain increased the efficiency of adenoviral transduction of cancer cell lines prompted us to study whether the increase in transduction efficiency observed in vitro and the partial reduction in liver transduction observed in vivo result in better transduction of cancer cells in vivo by AdTLRGDK compared with the nonmodified vector AdGFPLuc. 

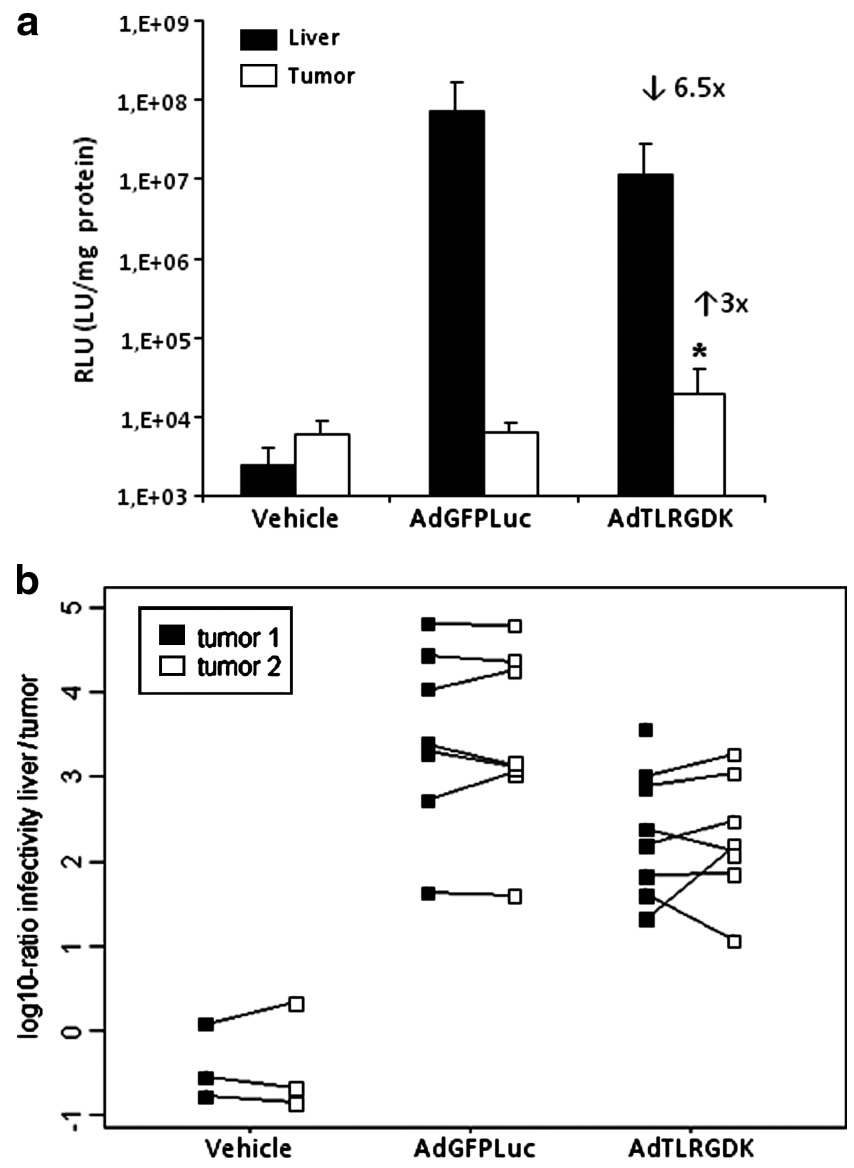

FIG. 4. (a) Tumor and liver luciferase expression in mice after systemic administration of fiber shaft-retargeted vector AdTLRGDK compared with nonmodified vector AdFGPLuc. The corresponding vector $\left(5 \times 10^{10} \mathrm{VP}\right)$ was injected intravenously into subcutaneously xenotransplanted BALB/c mice. Three days later, tumor and liver were harvested and luciferase activity and protein were quantified, as indicated in Materials and Methods. Results are shown as RLU [relative light units (mg protein) -1$] .{ }^{*} p \leq 0.05$, significantly different from nonmodified vector (Mann-Whitney test). (b) $\log _{10}$ ratio of liver to tumor transduction for recombinant vectors AdGFPLuc and AdTLRGDK was calculated for both tumors of each mouse with respect to liver. A line connects the value of the left flank tumor (solid squares) and right flank tumor (open squares) of each mouse.

With this aim we performed a biodistribution study in mice with subcutaneous BxPC3 tumors: $5 \times 10^{10}$ VP of unmodified vector AdGFPLuc or shaft-retargeted vector AdTLRGDK was injected via the tail vein. Three days after injection the luciferase activity in tumors and livers was quantified. The KKTK-to-RGDK replacement increased the transduction of BxPC3 tumors compared with tumors transduced with the nonmodified vector AdGFPLuc (Fig. 4). Logarithms of infectivity in liver and in tumor were calculated for both adenoviral vectors and then the liver-to-tumor $\log$ ratios were calculated for both vectors (Fig. 4b) The log ratio infectivity liver/tumor was higher for nonmodified vector than for AdTLRGDK ( $p$ value, 0.02); this result indicates that AdTLRGDK shows better targeting for tumors compared with the nonmodified vector AdGFPLuc.

\section{Discussion}

We studied the retargeting properties of the peptide RGD when used as a substitute for the KKT domain of the fiber shaft domain of Ad5. This site is the putative binding site for HSPG and therefore it could be exploited for presentation of tripeptide ligands such as RGD. In theory, this strategy could benefit simultaneously from the liver-detargeting properties of the KKTK domain deletion and the tumor-targeting properties of the RGD peptide, using at the same time a position far away from the knob, the fiber domain that needs extensive modifications to avoid blood factor binding (Shayakhmetov et al., 2005a). Our study focuses on transductional targeting/detargeting but not on particle targeting/ detargeting, which depends on particle clearance by extravasation in spleen and liver and interactions with other cell types such as macrophages, platelets, and erythrocytes that do not lead to gene expression. Transductional detargeting at the hepatocyte level is key to decreasing liver toxicity and it may allow an increase in administered dose to saturate these cellular interactions that are currently being elucidated.

Unlike the shaft mutations GATK and GAGA, that require fiber-complementing cells to be propagated (Smith et al., 2002; Bayo-Puxan et al., 2006), the RGDK shaft mutation can be propagated in 293 cells. The lack of deleterious effects on virus infectivity is likely due to the synonymous substitution of a positively charged $\mathrm{K}$ at position 91 for a positively charged R.

The RGD substitution of the KKTK fiber shaft domain resulted in increased tumor transduction efficiency compared with a nonmodified vector. It is important to note that AdTLRGDK transduced the CAR-low cell line L929 10 times better than the nonmodified vector, indicating that the tripeptide RGD located in the fiber shaft domain is exposed and could be recognized by cellular integrins. However, the fiber shaft mutation RGDK did not retain the same level of liver detargeting as the GATK mutation (Bayo-Puxan et al., 2006). This may be due to better preservation of the knobmediated, and hence blood factor, entry pathway. The increased tumor-to-liver ratio achieved with the RGD insertion at the shaft may be improved by additional modifications that prevent adenovirus binding to blood factors. Additional targeting ligands exposed at the HI-loop or located at other capsid proteins such as the hexon protein could also improve the tumor-targeting properties of shaft-mutated vectors. We are currently exploring the potential of such a virus with two RGDs, one at the shaft and another at the HI-loop of the knob (besides the natural one at the penton base). Our RGDK shaft mutation is unique in the sense that RGD is an exceptional case: a tripeptide that confers tumor infectivity. The potential to use this tripeptide for virus retargeting was demonstrated in viruses such as bacteriophages (Koivunen et al., 1993), hepatitis B (Chambers et al., 1996), and adenovirus (Wickham et al., 1997; Dmitriev et al., 1998) and many studies have demonstrated the value of the RGD tripeptide to target drugs toward tumors. Even our strategy is likely to be constrained because the KKTK domain at the shaft is not replaceable by large tumor-targeting ligands; we think that it is an important achievement because it capitalizes on the importance of RGD for the tumor-targeting field. A few other tripeptides, such as the NGR tumor-homing peptide 
(Arap et al., 1998), could also be used to replace the KKTK domain of adenovirus.

Apart from the interaction of blood factors with the fiber knob, the interaction of FX with the hexon protein contributes to the hepatotropism of adenovirus (Waddington et al., 2008). Future research projects will contemplate shaft-RGD viruses also detargeted from liver by means of hexon mutations. For simplicity, the extension of HVR5 of the hexon protein represents an attractive mutation to ablate FX interaction (Kalyuzhniy et al., 2008) and to use in combination with the RGDK mutation. Finally, we hope that the gains observed on transduction efficiency of tumor cells in vivo, using the RGDK mutation, in combination with genetic modifications to achieve cancer selective replication, intratumoral spread, and control of immune responses will increase the prospects of cancer virotherapy.

\section{Acknowledgments}

Funding was provided by grants BIO2005-08682-C03 and BIO2008-04692-C03-01 from the Ministerio de Educación y Ciencia, 2005 SGR 00727 from the Departament d'Universitats, Recerca i Societat de la Informació from the Generalitat de Catalunya, Theradpox EC 6th framework Contract 18700, and a grant from Mutua Madrileña. R.A. belongs to the Network of Cooperative Research on Cancer (C03-10), Instituto de Salud Carlos III of the Ministerio de Sanidad y Consumo, Government of Spain.

\section{Author Disclosure Statement}

The authors declare no competing financial interests.

\section{References}

Alemany, R., and Curiel, D.T. (2001). CAR-binding ablation does not change biodistribution and toxicity of adenoviral vectors. Gene Ther. 8, 1347-1353.

Arap, W., Pasqualini, R., and Ruoslahti, E. (1998). Cancer treatment by targeted drug delivery to tumor vasculature in a mouse model. Science 279, 377-380.

Baker, A.H., McVey, J.H., Waddington, S.N., Di Paolo, N.C., and Shayakhmetov, D.M. (2007). The influence of blood on in vivo adenovirus bio-distribution and transduction. Mol. Ther. 15, 1410-1416.

Bayo-Puxan, N., Cascallo, M., Gros, A., Huch, M., Fillat, C., and Alemany, R. (2006). Role of the putative heparan sulfate glycosaminoglycan-binding site of the adenovirus type 5 fiber shaft on liver detargeting and knob-mediated retargeting. J. Gen. Virol. 87, 2487-2495.

Bergelson, J.M., Cunningham, J.A., Droguett, G., Kurt-Jones, E.A., Krithivas, A., Hong, J.S., Horwitz, M.S., Crowell, R.L., and Finberg, R.W. (1997). Isolation of a common receptor for coxsackie B viruses and adenoviruses 2 and 5. Science 275, 1320-1323.

Chambers, M.A., Dougan, G., Newman, J., Brown, F., Crowther, J., Mould, A.P., Humphries, M.J., Francis, M.J., Clarke, B., Brown, A.L., and Rowlands, D. (1996). Chimeric hepatitis B virus core particles as probes for studying peptide-integrin interactions. J. Virol. 70, 4045-4052.

Dechecchi, M.C., Tamanini, A., Bonizzato, A., and Cabrini, G. (2000). Heparan sulfate glycosaminoglycans are involved in adenovirus type 5 and 2-host cell interactions. Virology 268, $382-390$.
Dechecchi, M.C., Melotti, P., Bonizzato, A., Santacatterina, M., Chilosi, M., and Cabrini, G. (2001). Heparan sulfate glycosaminoglycans are receptors sufficient to mediate the initial binding of adenovirus types 2 and 5. J. Virol. 75, 8772-8780.

Dmitriev, I., Krasnykh, V., Miller, C.R., Wang, M., Kashentseva, E., Mikheeva, G., Belousova, N., and Curiel, D.T. (1998). An adenovirus vector with genetically modified fibers demonstrates expanded tropism via utilization of a coxsackievirus and adenovirus receptor-independent cell entry mechanism. J. Virol. 72, 9706-9713.

Einfeld, D.A., Schroeder, R., Roelvink, P.W., Lizonova, A., King, C.R., Kovesdi, I., and Wickham, T.J. (2001). Reducing the native tropism of adenovirus vectors requires removal of both CAR and integrin interactions. J. Virol. 75, 11284-11291.

Kalyuzhniy, O., Di Paolo, N.C., Silvestry, M., Hofherr, S.E., Barry, M.A., Stewart, P.L., and Shayakhmetov, D.M. (2008). Adenovirus serotype 5 hexon is critical for virus infection of hepatocytes in vivo. Proc. Natl. Acad. Sci. U.S.A. 105, 5483-5488.

Koivunen, E., Gay, D.A., and Ruoslahti, E. (1993). Selection of peptides binding to the $\alpha_{5} \beta_{1}$ integrin from phage display library. J. Biol. Chem. 268, 20205-20210.

Kritz, A.B., Nicol, C.G., Dishart, K.L., Nelson, R., Holbeck, S., Von Seggern, D.J., Work, L.M., McVey, J.H., Nicklin, S.A., and Baker, A.H. (2007). Adenovirus 5 fibers mutated at the putative HSPG-binding site show restricted retargeting with targeting peptides in the HI loop. Mol. Ther. 15, 741-749.

Martin, K., Brie, A., Saulnier, P., Perricaudet, M., Yeh, P., and Vigne, E. (2003). Simultaneous CAR- and $\alpha_{v}$ integrin-binding ablation fails to reduce Ad5 liver tropism. Mol. Ther. 8, 485-494.

Mizuguchi, H., Koizumi, N., Hosono, T., Utoguchi, N., Watanabe, Y., Kay, M.A., and Hayakawa, T. (2001). A simplified system for constructing recombinant adenoviral vectors containing heterologous peptides in the HI loop of their fiber knob. Gene Ther. 8, 730-735.

Nemerow, G.R., and Stewart, P.L. (1999). Role of $\alpha_{\mathrm{v}}$ integrins in adenovirus cell entry and gene delivery. Microbiol. Mol. Biol. Rev. 63, 725-734.

Nicol, C.G., Graham, D., Miller, W.H., White, S.J., Smith, T.A., Nicklin, S.A., Stevenson, S.C., and Baker, A.H. (2004). Effect of adenovirus serotype 5 fiber and penton modifications on in vivo tropism in rats. Mol. Ther. 10, 344-354.

Parker, A.L., Waddington, S.N., Nicol, C.G., Shayakhmetov, D.M., Buckley, S.M., Denby, L., Kemball-Cook, G., Ni, S., Lieber, A., McVey, J.H., Nicklin, S.A., and Baker, A.H. (2006). Multiple vitamin K-dependent coagulation zymogens promote adenovirus-mediated gene delivery to hepatocytes. Blood 108, 2554-2561.

Parker, A.L., Mcvey, J.H., Doctor, J.H., Lopez-Franco, O., Waddington, S.N., Havenga, M.J., Nicklin, S.A., and Baker, A.H. (2007). Influence of coagulation factor zymogens on the infectivity of adenoviruses pseudotyped with fibers from subgroup D. J. Virol. 81, 3627-3631.

Rittner, K., Schreiber, V., Erbs, P., and Lusky, M. (2007). Targeting of adenovirus vectors carrying a tumor cell-specific peptide: In vitro and in vivo studies. Cancer Gene Ther. 14, 509-518.

Shayakhmetov, D.M., Eberly, A.M., Li, Z.Y., and Lieber, A. (2005a). Deletion of penton RGD motifs affects the efficiency of both the internalization and the endosome escape of viral particles containing adenovirus serotype 5 or 35 fiber knobs. J. Virol. 79, 1053-1061.

Shayakhmetov, D.M., Gaggar, A., Ni, S., Li, Z.Y., and Lieber, A. (2005b). Adenovirus binding to blood factors results in liver cell infection and hepatotoxicity. J. Virol. 79, 7478-7491. 
Smith, T., Idamakanti, N., Kylefjord, H., Rollence, M., King, L., Kaloss, M., Kaleko, M., and Stevenson, S.C. (2002). In vivo hepatic adenoviral gene delivery occurs independently of the coxsackievirus-adenovirus receptor. Mol. Ther. 5, 770-779.

Smith, T.A., Idamakanti, N., Marshall-Neff, J., Rollence, M.L., Wright, P., Kaloss, M., King, L., Mech, C., Dinges, L., Iverson, W.O., Sherer, A.D., Markovits, J.E., Lyons, R.M., Kaleko, M., and Stevenson, S.C. (2003a). Receptor interactions involved in adenoviral-mediated gene delivery after systemic administration in non-human primates. Hum. Gene Ther. 14, 15951604.

Smith, T.A., Idamakanti, N., Rollence, M.L., Marshall-Neff, J., Kim, J., Mulgrew, K., Nemerow, G.R., Kaleko, M., and Stevenson, S.C. (2003b). Adenovirus serotype 5 fiber shaft influences in vivo gene transfer in mice. Hum. Gene Ther. 14, 777-787.

Vigant, F., Descamps, D., Jullienne, B., Esselin, S., Connault, E., Opolon, P., Tordjmann, T., Vigne, E., Perricaudet, M., and Benihoud, K. (2008). Substitution of hexon hypervariable region 5 of adenovirus serotype 5 abrogates blood factor binding and limits gene transfer to liver. Mol. Ther. 16, 1474-1480. Waddington, S.N., Mcvey, J.H., Bhella, D., Parker, A.L., Barker, K., Atoda, H., Pink, R., Buckley, S.M., Greig, J.A., Denby, L., Custers, J., Morita, T., Francischetti, I.M., Monteiro, R.Q., Barouch, D.H., Van Rooijen, N., Napoli, C., Havenga, M.J., Nicklin, S.A., and Baker, A.H. (2008). Adenovirus serotype 5 hexon mediates liver gene transfer. Cell 132, 397-409.
Wickham, T.J., Mathias, P., Cheresh, D.A., and Nemerow, G.R. (1993). Integrins $\alpha_{\mathrm{v}} \beta_{3}$ and $\alpha_{\mathrm{v}} \beta_{5}$ promote adenovirus internalization but not virus attachment. Cell 73, 309-319.

Wickham, T.J., Tzeng, E., Shears, L.L., II, Roelvink, P.W., Li, Y., Lee, G.M., Brough, D.E., Lizonova, A., and Kovesdi, I. (1997). Increased in vitro and in vivo gene transfer by adenovirus vectors containing chimeric fiber proteins. J. Virol. 71, 82218229.

Xia, H., Anderson, B., Mao, Q., and Davidson, B.L. (2000). Recombinant human adenovirus: Targeting to the human transferrin receptor improves gene transfer to brain microcapillary endothelium. J. Virol. 74, 11359-11366.

Address correspondence to: Dr. Ramon Alemany Institut Català d'Oncologia Av Gran Via s/n km 2.7 L'Hospitalet de Llobregat 08907 Barcelona, Spain

E-mail: ralemany@iconcologia.net

Received for publication March 13, 2009; accepted after revision June 17, 2009.

Published online: August 6, 2009. 